\title{
Impacto de uma estratégia de manejo do excesso de peso infanto-juvenil
}

\author{
Impact of a management strategy on child and adolescent overweight
}

\author{
Lisete Griebeler Souza $\mathbb{D}^{1}$, Zilda Elizabeth de Albuquerque Santos $\mathbb{D}^{2}$, Mariur Gomes Beghetto $\mathbb{D}^{3}$, \\ Elza Daniel de Mello $\mathbb{D}^{1}$ \\ 1Faculdade de Medicina, Programa de Pós-Graduação em Saúde da Criança e do Adolescente. Universidade Federal do Rio Grande do Sul. \\ Porto Alegre, Rio Grande do Sul, Brasil. \\ 2 Faculdade de Medicina, Departamento de Nutrição, Universidade Federal do Rio Grande do Sul. \\ Porto Alegre, Rio Grande do Sul, Brasil. \\ ${ }^{3}$ Escola de Enfermagem, Universidade Federal do Rio Grande do Sul. Porto Alegre, Rio Grande do Sul, Brasil.
}

Como citar este artigo (How to cite this article):

Souza LG, Santos ZEA, Beghetto MG, Mello ED. Impacto de uma estratégia de manejo do excesso de peso infanto-juvenil (Impact of a management strategy on child and adolescent overweight). Sci Med. 2019;29(3):e33486. https://doi.org/10.15448/1980-6108.2019.3.33486

\section{RESUMO}

OBJETIVOS: Avaliar, através de parâmetros antropométricos e bioquímicos, o impacto de uma estratégia de manejo do excesso de peso implantada em um ambulatório de obesidade infanto-juvenil de um hospital público.

MÉTODOS: Análise retrospectiva de uma coorte, realizada a partir de fichas de 206 crianças e adolescentes com diagnóstico de obesidade ou sobrepeso, com idade entre 5 a 17 anos, atendidos entre 2010 e 2014 com periodicidade média de três meses durante um ano. A estratégia avaliada consistiu em aconselhamento, com orientações visando redução do tempo de atividade sedentária e aumento de atividade física e orientações nutricionais para escolhas alimentares mais saudáveis. Foram avaliados parâmetros antropométricos (índice de massa corporal (IMC), escore z de IMC por idade, circunferências da cintura (CC) e do quadril (CQ), razão cintura quadril (RCQ)) e bioquímicos (glicemia de jejum, insulinemia de jejum, índice do Homeostasis Model Assessment, colesterol total (CT), lipoproteína de alta densidade (HDL-c), lipoproteína de baixa densidade (LDL-c), triglicerídeos (TG) no início, aos 6 e aos 12 meses de acompanhamento. As análises estatísticas foram realizadas no programa IBM SPSS Statistics 22.0, com aplicação do modelo de Equações de Estimativas Generalizadas.

RESULTADOS: Em 12 meses, houve redução significativa do IMC, do escore $\mathrm{z}$ do IMC/idade, do percentual de indivíduos com escore $\mathrm{z}$ de IMC / idade $\geq+2$, da CC, do percentual de indivíduos com CC > p90 e da RCQ (p<0,001). Os níveis séricos de glicemia e insulina não apresentaram redução significativa, apenas uma tendência, assim como os de CT, LDL-c e TG, porém os níveis de HDL-c tiveram um aumento significativo $(\mathrm{p}=0,035)$.

CONCLUSÕES: Esta estratégia de manejo do excesso de peso infanto-juvenil, baseada em aconselhamento, possibilitou redução significativa dos níveis de excesso de peso, embora os parâmetros bioquímicos não tenham sido reduzidos na mesma proporção.

UNITERMOS: Obesidade pediátrica; educação alimentar e nutricional; perda de peso

\section{ABSTRACT}

AIMS: To evaluate, through anthropometric and biochemical parameters, the impact of an overweight management strategy implemented in an outpatient clinic for childhood obesity in a public hospital.

METHODS: Retrospective analysis of a cohort of 206 children and adolescents with a diagnosis of obesity or overweight, aged between 5 and 17 years, attended between 2010 and 2014 with an average period of three months during one year. The strategy evaluated consisted of counseling, with guidelines aimed at reducing sedentary activity time and increased physical activity and nutritional guidelines for healthier food choices. Were evaluated anthropometric parameters (body mass index, z-escore of body mass index by age, waist circumference, hip circumference and waist hip ratio) and biochemistry (fasting glucose, fasting insulinemia, homeostasis model assessment, total cholesterol (CT), high density lipoprotein (HDL-c), low density lipoprotein (LDL-c), triclycerides (TG) at the baseline, at 6 and 12 months of follow-up. Statistical analyzes were performed in the IBM SPSS Statistics 22.0, using the Generalized Estimating Equations model.

RESULTS: In 12 months, there was a significant reduction in BMI, Z score of BMI/age, percentage of individuals with BMI/age $\mathrm{z}$ score $\geq+2$, WHR, percentage of individuals with $\mathrm{WC}>\mathrm{p} 90$ and $\mathrm{HC}(\mathrm{p}<0,001)$. Serum glycemia and insulin levels did not show significant reduction, only a trend, as did TC, LDL-c and TG, but HDL-c levels had a significant increase $(\mathrm{p}=0.035)$.

CONCLUSIONS: This strategy of management of childhood and adolescent overweight, based on counseling, made it possible to reduce significantly the levels of overweight, although the biochemical parameters were not reduced in the same proportion.

KEYWORDS: Pediatric obesity; food and nutrition education; weight loss. 
Abreviaturas: AmO, Ambulatório de Obesidade infanto-juvenil; CC, Circunferência da Cintura; CQ, Circunferência do Quadril; CT, Colesterol Total; DM2, Diabetes Mellitus tipo 2; GEE, Equações de Estimativas Generalizadas; HCPA, Hospital de Clínicas de Porto Alegre; HDL-c, Lipoproteína de Alta Densidade; HOMA, Homeostasis Model Assessment; IMC, Índice de Massa Corporal; LDL-c, Lipoproteína de Baixa Densidade; POEMGMV, Pediatric Obesity Empowerment Model Group Medical Visits; RCQ, Razão Cintura Quadril; SISVAN, Sistema de Vigilância Alimentar e Nutricional do Ministério da Saúde; SPSS, Statistical Package for Social Sciences; TCOCT, The Children's Obesity Clinic's Treatment; TG, Triglicerídeos.

\section{INTRODUÇÃO}

Segundo a Organização Mundial de Saúde, em 2016 em todo o mundo, 41 milhões de crianças com idade inferior a 5 anos estavam obesas e mais de 340 milhões de crianças e adolescentes com idade entre 5 e 19 anos estavam acima do peso ou obesas. A prevalência de sobrepeso e obesidade entre crianças e adolescentes aumentou de 4\% em 1975 para pouco mais de $18 \%$ em 2016. Estes dados tornam a obesidade infantil um dos maiores desafios de saúde pública do século XXI. No Brasil, dados da Pesquisa de Orçamento Familiar, divulgada pelo Instituto Brasileiro de Geografia e Estatística, mostraram que $16,6 \%$ dos meninos e $11,8 \%$ das meninas entre 5 e 9 anos de idade estavam obesas $[1,2]$.

A criança com obesidade tem risco maior de permanecer nesta condição quando adulta, se comparada à criança eutrófica, e o tempo de duração da obesidade está diretamente relacionado à morbimortalidade por doenças cardiovasculares. Altos níveis de índice de massa corporal (IMC) entre crianças e adolescentes estão associados a níveis adversos de lipídios, insulina e pressão arterial. Em torno de 39\% das crianças com excesso de peso e $59 \%$ das com obesidade, apresentam pelo menos dois destes fatores de risco para doenças cardiovasculares (hiperinsulinemia, dislipidemia e hipertensão) $[1,3]$.

Várias estratégias de intervenção para manejo da obesidade infanto-juvenil já foram utilizadas, tanto com atuação na alimentação (como dietas hipocalóricas, dietas hipoglicídicas e hiperproteicas, dietas de reduzida carga glicêmica ou dieta do semáforo), quanto na atividade física, na mudança comportamental e do ambiente familiar, além de projetos comunitários. Apesar de não estar definido um modelo padrão na abordagem deste problema, as intervenções em ambiente familiar de base comportamental, que incorporam modificações na alimentação e na atividade física, parecem ser as mais efetivas no controle do peso corporal em crianças e adolescentes [4].
Uma vez que a obesidade é uma doença crônica que determina inúmeras comorbidades e deve ser tratada de forma eficaz e o mais precocemente possível e que o manejo da obesidade é difícil, especialmente na fase infanto-juvenil, é importante validar novas estratégias que possam proporcionar resultados positivos no manejo do excesso de peso nesta faixa etária. O objetivo deste estudo foi avaliar, através de parâmetros antropométricos e bioquímicos, o impacto de uma estratégia de manejo do excesso de peso implantada em um ambulatório de obesidade infanto-juvenil de um hospital público em um ano de atendimento.

\section{MÉTODOS}

O presente estudo caracteriza-se como uma análise retrospectiva de uma coorte e foi realizado em um banco de dados organizado a partir das fichas preenchidas por ocasião das consultas das crianças e adolescentes atendidas no ambulatório de obesidade infanto-juvenil de um hospital público entre 2010 e 2014. Foram incluídos indivíduos de ambos os sexos, com idade entre 5 e 17 anos, com diagnóstico de obesidade ou sobrepeso que compareceram às consultas com periodicidade média de três meses durante um ano e que realizaram exames bioquímicos no início, no meio e no final deste período. Foram excluídos os portadores de doença mental ou psicológica, aqueles com excesso de peso decorrente de síndromes genéticas e/ou de uso de medicamentos e os que não tinham o número e período de consultas e exames necessários.

As variáveis estudadas foram índice de massa corporal (IMC), escore z de IMC por idade, circunferências da cintura (CC) e do quadril (CQ), razão cintura quadril (RCQ), glicemia de jejum, insulinemia de jejum, índice do Homeostasis Model Assessment (HOMA), colesterol total (CT), lipoproteína de alta densidade (HDL-c), lipoproteína de baixa densidade (LDL-c), triglicerídeos (TG) e uso de metformina.

A estratégia empregada para o manejo do excesso de peso era baseada em aconselhamento, focada no estímulo à mudanças de hábito de vida, com orientações visando a redução do tempo de atividade sedentária e incentivo à realização de atividades físicas (brincadeiras, jogos, atividades domésticas, deslocamentos e, se possível, atividades orientadas e sistemáticas) e orientações nutricionais, tais como aumento da ingestão de água, redução de refrigerantes, doces e frituras, atenção ao tipo e tamanho das porções de alimentos e aos horários, frequência e local das refeições $[5,6]$. 
Na primeira consulta eram verificados os hábitos alimentares e de atividade física e era realizada avaliação antropométrica e solicitação de exames laboratoriais. Em todas as consultas, com duração aproximada de 30 minutos, os pacientes eram atendidos individualmente, acompanhados por um responsável. As consultas eram realizadas preferencialmente pelo mesmo profissional (médico, nutricionista, enfermeiro ou acadêmicos de medicina, nutrição ou enfermagem), que estabelecia, em conjunto com o paciente, dois a três objetivos de mudanças na alimentação e atividade física, que seriam avaliados no encontro seguinte. Em todas as consultas eram realizadas avaliação antropométrica, dos hábitos alimentares e da rotina de atividade física, para verificar a adesão às combinações anteriores e os resultados obtidos. Os exames bioquímicos eram solicitados a cada seis meses, em média.

Para avaliação dos hábitos alimentares era aplicado uma adaptação simplificada da história alimentar, inquérito dietético utilizado na prática clínica com o objetivo de conhecer os hábitos alimentares, capaz de estimar a ingestão habitual qualitativa [7].

$\mathrm{Na}$ avaliação antropométrica, foram realizadas as seguintes medidas: peso, altura, CC e CQ. A aferição do peso corporal era feita com uso de avental hospitalar, em balança antropométrica digital com capacidade para $150 \mathrm{~kg}$ e a da altura, por meio de estadiômetro de parede, conforme técnicas padronizadas pelo Sistema de Vigilância Alimentar e Nutricional do Ministério da Saúde [8]. Os dados de peso e altura serviam para cálculo do IMC através da fórmula: $\mathrm{IMC}=$ peso $(\mathrm{kg}) /$ altura $^{2}(\mathrm{~m})$, colocado na curva de IMC/idade da Organização Mundial de Saúde para verificação de adequação. Para definição de sobrepeso e obesidade, foram utilizados os padrões do Sistema de Vigilância Alimentar e Nutricional do Ministério da Saúde, que classifica como sobrepeso os indivíduos com escore $\mathrm{z}$ do IMC por idade $\geq+1$ e $<+2$ e como obesos os com escore $\mathrm{z}$ do IMC por idade $\geq+2$ [8]. A medida da CC era realizada com uma fita inelástica, no ponto médio entre a borda inferior da última costela e a borda superior da crista ilíaca, e a da CQ, na região de maior perímetro entre a cintura e a coxa. Os dados de CC e CQ serviam para o cálculo da RCQ. O ponto de corte adotado para a CC foi $>$ p90 para sexo e idade, de acordo com Freedman et al., no Bogalusa Heart Study [9].

Os exames bioquímicos eram realizados no laboratório da própria instituição. Os valores de referência para lipídios na infância e adolescência utilizados foram os recomendados pela V Diretriz brasileira de dislipidemia e prevenção da aterosclerose, que con- sidera inadequados valores de $\mathrm{CT} \geq 170 \mathrm{mg} / \mathrm{dl}$, LDL-c $\geq 130 \mathrm{mg} / \mathrm{dl}, \mathrm{HDL}-\mathrm{c} \leq 45 \mathrm{mg} / \mathrm{dl} \mathrm{e} \mathrm{TG} \geq 130 \mathrm{mg} / \mathrm{dl}$ [10]. $\mathrm{O}$ ponto de corte para insulina de jejum adotado foi o descrito pela Sociedade Brasileira de Pediatria, que considera valores de insulinemia basal acima de $15 \mu \mathrm{U} / \mathrm{ml}$ característico de resistência à insulina [11]. Os dados de glicemia e insulina foram utilizados para o cálculo do índice HOMA, e o ponto de corte foi o valor de 3,16 proposto por Keskin et al. [12]. Os jovens com obesidade severa, histórico familiar de diabetes mellitus tipo 2, insulina de jejum e índice HOMA alterados, tiveram a prescrição de metformina $500 \mathrm{mg} 2$ vezes ao dia, protocolo semelhante ao usado no estudo de Brufani et al. [13].

Este estudo foi aprovado pelo comitê de ética do Hospital de Clínicas de Porto Alegre, com parecer número 1496802 e na Plataforma Brasil, com CAAE 53876716.0.0000.5327. As análises estatísticas foram realizadas no programa IBM SPSS Statistics 22.0. As variáveis quantitativas simétricas (IMC, escore $\mathrm{z}$ de IMC por idade, CC, CQ, RCQ, glicemia, CT, HDL-c, LDL-c, TG) foram descritas por média e desvio padrão, as quantitativas assimétricas (insulina, HOMA) por mediana e percentis 25 e 75 e as categóricas (escore $\mathrm{z}$ de IMC por idade $\geq+2, \mathrm{CC}>\mathrm{p} 90$, glicemia $>100 \mathrm{mg} / \mathrm{dl}$, insulina $>15 \mu \mathrm{U} / \mathrm{ml}$, HOMA $>$ 3,16 , uso de metformina, CT $>170 \mathrm{mg} / \mathrm{dl}$, HDL-c $<$ $45 \mathrm{mg} / \mathrm{dl}, \mathrm{LDL}-\mathrm{c}>130 \mathrm{mg} / \mathrm{dl}, \mathrm{TG}>130 \mathrm{mg} / \mathrm{dl}$ ) por frequências absolutas e relativas. Para as comparações dos parâmetros ao longo do tempo, foi aplicado o modelo de equações de estimativas generalizadas, com ajuste de Bonferroni [14]. O modelo linear foi utilizado para as variáveis de distribuição simétrica, o de gamma para as assimétricas e o binário para as variáveis categóricas. Para avaliar a associação entre as variações dos parâmetros quantitativos ao longo do tempo, os testes da correlação de Pearson (distribuição simétrica) ou de Spearman (distribuição assimétrica) foram aplicados. O nível de significância adotado foi de $5 \%(\mathrm{p}<0,05)$.

\section{RESULTADOS}

Das 602 crianças e adolescentes atendidas no ambulatório de obesidade infanto-juvenil, foram avaliadas 206 crianças e adolescentes entre 5 e 17 anos de idade, com média de 10,6 $6 \pm 3,0$ anos, dos quais 112 $(54,4 \%)$ eram do sexo masculino. Do total, 180 (87\%) tinham pai ou mãe com IMC maior ou igual a $25 \mathrm{~kg} / \mathrm{m}^{2}$ (excesso de peso) e 107 (51,7\%) tinham pai e mãe com excesso de peso. O escore $\mathrm{z}$ de IMC por idade variava de 1,28 a 8,67 com média $3,49 \pm 1,21$. 
No início do estudo, 96,1\% das crianças e adolescentes apresentavam obesidade, $85,9 \%$ tinham a CC maior do que o percentil 90 para sexo e idade, $68,4 \%$ insulina de jejum maior que $15 \mu \mathrm{U} / \mathrm{ml}, 69,8 \% \mathrm{HOMA}$ maior que 3,16 e $77,2 \%$ HDL-c menor do que $45 \mathrm{mg} / \mathrm{dl}$. Os dados antropométricos e bioquímicos da amostra no início do estudo estão descritos na Tabela 1.

A evolução dos dados antropométricos e bioquímicos dos indivíduos submetidos a esse manejo de excesso de peso em seis meses e um ano de atendimento está descrita na Tabela 2. Em 12 meses, houve uma redução significativa no IMC, no escore $Z$ de IMC por idade, no número de indivíduos com escore $\mathrm{z}$ de IMC por idade $\geq+2$, na $C C$, no número de indivíduos com CC maior que percentil 90 e na RCQ. Com relação à $\mathrm{CQ}$, houve redução significativa nos primeiros 6 meses, mas esta não se manteve dos 6 aos 12 meses.

Quanto ao perfil glicêmico, neste período de acompanhamento, não houve redução dos níveis médios de glicemia e a redução da insulina não foi significativa, no entanto, o percentual de indivíduos que apresentavam glicemia $>$ que $100 \mathrm{mg} / \mathrm{dl}$ passou de $12,1 \%$ no início do estudo para $9 \%$ no final, assim como de insulina de jejum $>15 \mu \mathrm{U} / \mathrm{ml}$, que passou de $68,4 \%$ para $65,6 \%$. O índice HOMA apresentou uma redução significativa em 6 meses, mas esta redução não se manteve dos 6 aos 12 meses.
Tabela 1. Caracterização da amostra no início do estudo quanto as variáveis analisadas

\begin{tabular}{|c|c|}
\hline Variáveis & $\mathrm{n}=\mathbf{2 0 6}$ \\
\hline IMC $\left(\mathrm{kg} / \mathrm{m}^{2}\right)^{*}$ & $29,6 \pm 5,1$ \\
\hline Escore z IMC por idade* & $3,49 \pm 1,21$ \\
\hline Escore $\mathrm{z}$ IMC por idade $\geq+2^{+}$ & $198(96,1)$ \\
\hline $\mathrm{CC}(\mathrm{cm})^{*}$ & $88,7 \pm 11,8$ \\
\hline $\mathrm{CC}>\mathrm{p} 90^{+}$ & $177(85,9)$ \\
\hline $\mathrm{CQ}(\mathrm{cm})^{*}$ & $97,3 \pm 13,2$ \\
\hline $\mathrm{RCQ}^{*}$ & $0,91 \pm 0,07$ \\
\hline Glicemia $(\mathrm{mg} / \mathrm{dl})^{*}$ & $90,7 \pm 6,7$ \\
\hline Glicemia $>100 \mathrm{mg} / \mathrm{dl}^{+}$ & $25(12,1)$ \\
\hline Insulina $(\mu \mathrm{U} / \mathrm{ml})^{\ddagger}$ & $20,2(13,3-27,4)$ \\
\hline Insulina $>15 \mu \mathrm{U} / \mathrm{ml}^{+}$ & $141(68,4)$ \\
\hline $\mathrm{HOMA}^{\ddagger}$ & $4,5(2,8-6,5)$ \\
\hline $\mathrm{HOMA}>3,16^{+}$ & $143(69,8)$ \\
\hline Uso de Metformina ${ }^{\ddagger}$ & $0(0,0)$ \\
\hline $\mathrm{CT}(\mathrm{mg} / \mathrm{dl})^{*}$ & $161 \pm 32,6$ \\
\hline $\mathrm{CT}>170 \mathrm{mg} / \mathrm{dl}^{+}$ & $80(38,8)$ \\
\hline HDL-c (mg/dl)* & $39,7 \pm 8,5$ \\
\hline HDL-c $<45 \mathrm{mg} / \mathrm{dl}^{+}$ & $159(77,2)$ \\
\hline LDL-c (mg/dl)* & $100 \pm 27,0$ \\
\hline LDL-C $>130 \mathrm{mg} / \mathrm{dl}^{+}$ & $29(14,1)$ \\
\hline TG (mg/dl)* & $108 \pm 54,6$ \\
\hline $\mathrm{TG}>130 \mathrm{mg} / \mathrm{dl}^{+}$ & $60(29,1)$ \\
\hline
\end{tabular}

* média \pm desvio padrão; ${ }^{\dagger} \mathrm{n}(\%) ;{ }^{\ddagger}$ mediana (amplitude interquartil). CC: Circunferência da Cintura: CQ: Circunferência do Quadril: CT: Colesterol Total, HDL-†キc: Lipoproteína de Alta Densidade; HOMA: Homeostasis Model Assessmen IMC: Índice de Massa Corporal; LDL-c: Lipoproteína de Baixa Densidade; RCQ: Razão Cintura Quadril; TG: Triglicerídeos.

Tabela 2. Evolução dos dados antropométricos e bioquímicos ao longo do estudo.

\begin{tabular}{|c|c|c|c|c|}
\hline Variáveis & Basal $(n=206)$ & 6 meses $(n=201)$ & 12 meses $(n=154)$ & p \\
\hline \multicolumn{5}{|l|}{ Avaliação antropométrica } \\
\hline $\mathrm{IMC}\left(\mathrm{kg} / \mathrm{m}^{2}\right)^{*}$ & $29,6 \pm 0,4^{b}$ & $28,8 \pm 0,4^{\mathrm{a}}$ & $28,6 \pm 0,5^{\mathrm{a}}$ & $<0,001$ \\
\hline Escore z IMC por idade* & $3,49 \pm 0,08^{c}$ & $3,18 \pm 0,08^{b}$ & $2,86 \pm 0,08^{a}$ & $<0,001$ \\
\hline Escore $\mathrm{z} I \mathrm{IMC} \geq+2^{+}$ & $198(96,1)^{c}$ & $177(88,1)^{b}$ & $120(77,9)^{\mathrm{a}}$ & $<0,001$ \\
\hline Escore $\mathrm{z}+1 \leq \mathrm{IMC}<+2^{+}$ & $8(3,9)^{\mathrm{a}}$ & $24(11,9)^{b}$ & $31(20,1)^{c}$ & $<0,001$ \\
\hline $\mathrm{CC}(\mathrm{cm})^{*}$ & $88,7 \pm 0,8^{b}$ & $86,7 \pm 0,8^{a}$ & $86,2 \pm 1,0^{\mathrm{a}}$ & $<0,001$ \\
\hline $\mathrm{CC}>\mathrm{P} 0^{+}$ & $177(85,9)^{c}$ & $152(76,4)^{b}$ & $93(64,6)^{\mathrm{a}}$ & $<0,001$ \\
\hline $\mathrm{CQ}(\mathrm{cm})^{*}$ & $97,3 \pm 0,9^{b}$ & $96,3 \pm 0,9^{a}$ & $97,3 \pm 1,1^{\text {ab }}$ & 0,004 \\
\hline RCQ* & $0,91 \pm 0,005^{c}$ & $0,90 \pm 0,004^{b}$ & $0,89 \pm 0,004^{a}$ & $<0,001$ \\
\hline \multicolumn{5}{|l|}{ Perfil glicêmico } \\
\hline Glicemia (mg/dl)* & $90,7 \pm 0,5$ & $89,9 \pm 0,5$ & $90,2 \pm 0,5$ & 0,151 \\
\hline Glicemia $>100 \mathrm{mg} / \mathrm{dl}^{+}$ & $25(12,1)$ & $15(7,5)$ & $14(9,0)$ & 0,143 \\
\hline Insulina $(\mu \mathrm{U} / \mathrm{ml})^{*}$ & $22,7 \pm 1,0$ & $20,6 \pm 0,8$ & $21,8 \pm 1,2$ & 0,066 \\
\hline Insulina $>15 \mu \mathrm{U} / \mathrm{ml}^{+}$ & $141(68,4)$ & $131(65,2)$ & $101(65,6)$ & 0,596 \\
\hline HOMA* & $5,11 \pm 0,23^{b}$ & $4,59 \pm 0,18^{a}$ & $4,86 \pm 0,28^{a b}$ & 0,047 \\
\hline $\mathrm{HOMA}>3,16^{+}$ & $143(69,8)$ & $143(71,5)$ & $106(68,8)$ & 0,769 \\
\hline Uso de Metformina ${ }^{+}$ & $0(0,0)^{\mathrm{a}}$ & $103(51,2)^{b}$ & $76(49,4)^{\mathrm{b}}$ & $<0,001$ \\
\hline \multicolumn{5}{|l|}{ Perfil lipídico } \\
\hline $\mathrm{CT}(\mathrm{mg} / \mathrm{dl}) *$ & $161 \pm 2,3$ & $160 \pm 2,3$ & $157 \pm 2,5$ & 0,148 \\
\hline $\mathrm{CT}>170 \mathrm{mg} / \mathrm{dl}^{+}$ & $80(38,8)$ & $68(33,8)$ & $50(32,5)$ & 0,178 \\
\hline HDL-c (mg/dl)* & $39,7 \pm 0,6^{a}$ & $40,9 \pm 0,7^{b}$ & $41,1 \pm 0,7^{b}$ & 0,035 \\
\hline $\mathrm{HDL}-\mathrm{C}<45 \mathrm{mg} / \mathrm{dl}^{+}$ & $159(77,2)$ & $147(73,1)$ & $108(70,1)$ & 0,182 \\
\hline LDL-C (mg/dl)* & $100 \pm 1,9$ & $98,7 \pm 1,9$ & $96,0 \pm 2,0$ & 0,062 \\
\hline $\mathrm{LDL}-\mathrm{C}>130 \mathrm{mg} / \mathrm{ml}^{+}$ & $29(14,1)$ & $23(11,4)$ & $15(9,7)$ & 0,238 \\
\hline TG $(\mathrm{mg} / \mathrm{dl}) *$ & $108 \pm 3,8$ & $104 \pm 3,7$ & $100 \pm 4,4$ & 0,139 \\
\hline $\mathrm{TG}>130 \mathrm{mg} / \mathrm{dl}^{+}$ & $60(29,1)$ & $46(22,9)$ & $37(24,0)$ & 0,155 \\
\hline
\end{tabular}


No perfil lipídico, ao longo do estudo, a redução dos níveis de CT, LDL-c e TG não foi significativa, porém o percentual de indivíduos que apresentavam $\mathrm{CT}>170 \mathrm{mg} / \mathrm{dl}$ passou de $38,8 \%$ no início para $32,5 \%$ no final, assim como de LDL-c $>130 \mathrm{mg} / \mathrm{dl}$, que passou de $14,1 \%$ para $9,7 \%$, e TG $>130 \mathrm{mg} / \mathrm{dl}$, que foi de $29,1 \%$ para $24 \%$. Já os níveis de HDL-c tiveram aumento significativo em 12 meses, e o percentual de indivíduos com HDL-c $<45 \mathrm{mg} / \mathrm{dl}$ baixou de $77,2 \%$ para $70,1 \%$. Não foram utilizados medicamentos para controle de dislipidemia, sendo estes resultados atribuídos somente à estratégia utilizada, com melhora dos hábitos de vida e redução de peso.

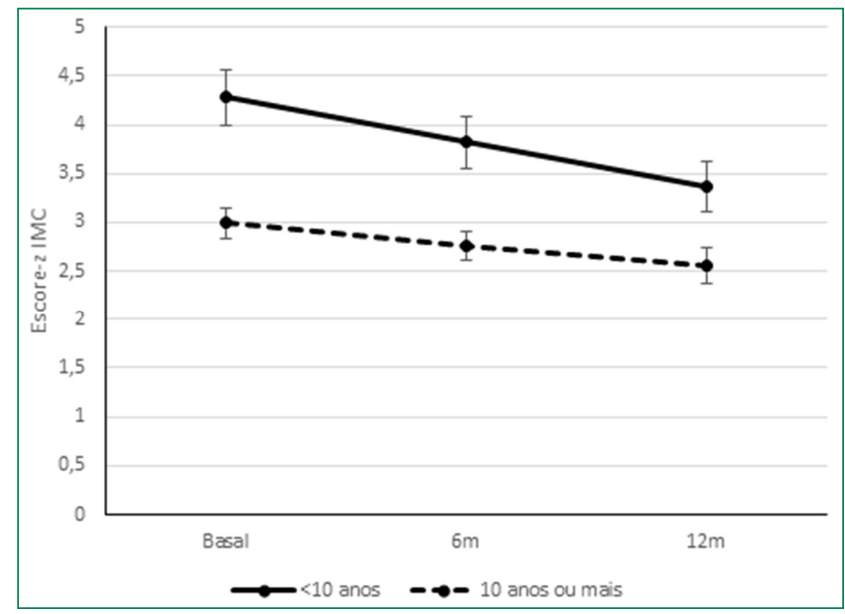

Figura 1. Interação entre faixa etária e redução de escore z de IMC por idade em 1 ano de acompanhamento $(p=0,001)$. O teste utilizado foi o modelo de equações de estimativas generalizadas complementado pelo teste de Bonferroni. (IMC: Índice de Massa Corporal).

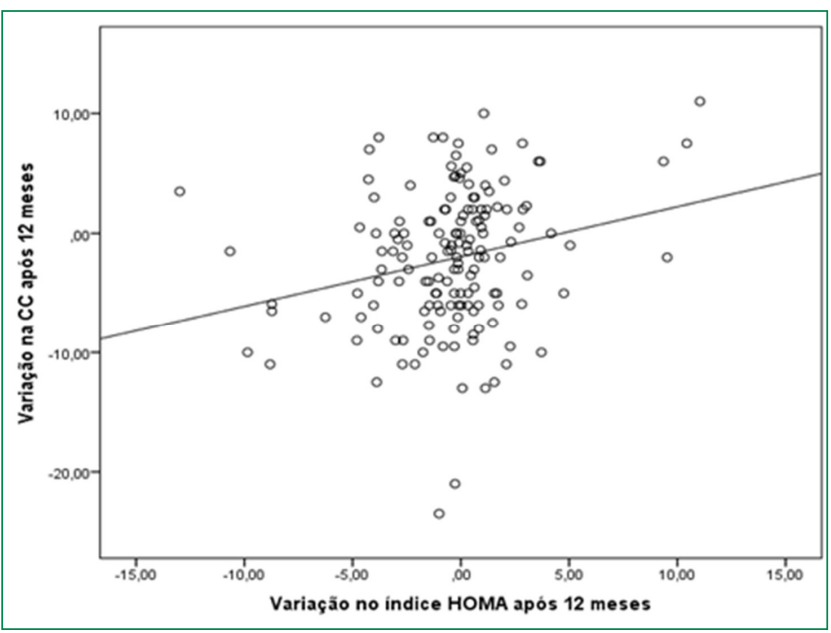

Figura 2. Correlação entre circunferência da cintura e Homesostasis Model Assessment ao longo do seguimento de 1 ano $(r=0,219 ; p=0,01)$. CC: Circunferência da Cintura; HOMA: Homesostasis Model Assessment
Dividindo a amostra, segundo os critérios do Sistema de Vigilância Alimentar e Nutricional do Ministério da Saúde, em crianças (até 10 anos incompletos) e adolescentes (de 10 a 20 anos) e verificando a interação entre as faixas etárias e os resultados dos parâmetros ao longo do tempo, observou-se interação significativa apenas em Escore $\mathrm{z}$ de IMC por idade $(p<0,001)$ [6]. Conforme pode ser observado na

Figura 1, as crianças apresentaram maior redução no Escore $\mathrm{z}$ de IMC por idade do que os adolescentes em 1 ano de seguimento, reduzindo $21,5 \%$ enquanto os adolescentes reduziram 16,8\%. Outro achado importante ao avaliar as faixas etárias, é que as crianças apresentavam valores de escore $\mathrm{z}$ de IMC por idade maiores do que os adolescentes. Não foram encontradas diferenças significativas entre os sexos para nenhum dos parâmetros.

Avaliando a correlação entre as variações de CC e de índice HOMA, foi constatada uma correlação positiva significativa, apesar de fraca, como mostra a Figura 2.

Após a primeira consulta, 103 indivíduos $(51,2 \%)$ passaram a utilizar metformina e aos 12 meses, $76(49,4 \%)$ continuavam usando, mas não houve diferença no IMC e nem redução significativa do percentual de indivíduos com índice HOMA maior do que 3,16 nos que utilizaram a medicação comparados aos que não utilizaram. No entanto, nos que utilizaram o medicamento, o percentual de indivíduos que apresentavam HOMA maior do que 3,16 passou de $73,8 \%$ para $68,4 \%$ dos 6 aos 12 meses de acompanhamento e nos que não utilizaram, permaneceu em torno de $69 \%$.

\section{DISCUSSÃO}

Poucas intervenções realizadas com crianças e adolescentes produzem emagrecimento significativo a longo prazo, mas algumas estratégias têm mostrado evidências do benefício de uma abordagem focada no estilo de vida, incluindo uma dieta baseada na qualidade dos alimentos e incentivo à prática de atividade física [15].

Andela et al. [16] realizaram uma metanálise para verificar a eficácia e a segurança de programas de dieta com muito baixas calorias para perda de peso de crianças e adolescentes de 5 a 18 anos com obesidade. Encontraram que os resultados relacionados ao peso melhoraram significativamente após a intervenção em todos os estudos, porém os detalhes com relação aos efeitos adversos de uma restrição calórica severa nesta faixa etária foram limitados. Quatorze estudos não 
relataram a monitoração dos efeitos adversos gerais, mas seis estudos relataram a presença de efeitos adversos, incluindo fadiga, fome, tontura postural, náuseas, cãibras musculares, mau hálito e cefaleia, e um estudo que monitorou a função cardíaca, detectou que a perda de peso a curto prazo com restrição severa resultou em algumas alterações significativas, como diminuição da frequência cardíaca e arritmias potenciais, além de 2 casos mais graves. Com relação a crescimento, a maioria dos estudos não encontrou redução significativa, porém as intervenções eram curtas, em um estudo mais longo, a velocidade de crescimento diminuiu. Estes achados não permitem concluir sobre a segurança de intervenções com restrição severa para crianças e adolescentes.

Este estudo, com uma estratégia de manejo baseada em estímulo a mudanças de hábito de vida, nutricionais e de atividade física, mostrou resultados efetivos e seguros no tratamento da obesidade infanto-juvenil, produzindo redução significativa nos índices antropométricos dos indivíduos em um ano de acompanhamento, tanto no IMC como na $\mathrm{CC}$ e RCQ. Este é um achado importante, considerando-se que a obesidade infantil é um dos maiores desafios de saúde atual e deve ser tratada antes do aparecimento das comorbidades associadas a obesidade, visando reduzir o seu impacto econômico e social [17].

Zolotarjova et al. realizaram uma revisão em 2018, avaliando 16 estudos entre 1995 e 2017 com jovens com obesidade mórbida, de 4 a 18 anos de idade, submetidos a tratamentos multidisciplinares e encontraram que todos os estudos tiveram redução do IMC ou escore z de IMC e, quando medidos, redução dos fatores de risco cardiovascular. Concluíram que não há consenso sobre o tratamento da obesidade infantil, mas que a maioria das estratégias de intervenção que obtém resultados são semelhantes a estratégia utilizada neste estudo, que inclui alteração da atividade física com educação nutricional e modificação de comportamento, com envolvimento familiar, centrada no reforço positivo e definição de metas. Assim como neste estudo, na maioria dos artigos avaliados, os resultados foram maiores nas idades mais jovens, porém obtiveram melhores resultados nos meninos, enquanto neste estudo não houve diferença significativa entre os sexos [18].

Most et al. [19], em uma clínica dinamarquesa de tratamento da obesidade pediátrica, adotando o protocolo de tratamento The Children's Obesity Clinic's Treatment, que utiliza um plano de tratamento individualizado, com aconselhamento em conjunto com a família, também encontraram diferença significativa no IMC após 1 e 2 anos e também obtiveram melhores resultados nos meninos.

Assim como Serodio et al., que determinaram a eficácia de um programa de manejo de peso pediátrico para redução da obesidade semelhante a este, financiado pelo governo no Canadá, encontraram uma redução significativa no percentil de IMC e diminuição da prevalência da obesidade de $96,7 \%$ para $87,5 \%$. Os pacientes que tiveram tempos mais longos de tratamento, experimentaram reduções maiores na obesidade, mostrando que a participação do paciente a longo prazo pode ser necessária para suportar melhor os pacientes de controle de peso pediátricos [20]. Avaliando o presente estudo, pode-se acreditar que com maior tempo de acompanhamento os resultados seriam ainda melhores, pois em 1 ano já foram encontradas reduções significativas dos níveis de obesidade.

Neste estudo, além dos resultados positivos na redução de IMC e escore $\mathrm{z}$ de IMC, observou-se redução da obesidade abdominal, através da avaliação da $\mathrm{CC}$, e melhora em alguns parâmetros bioquímicos, como HDL-c e HOMA. Geller et al. [21], em estudo de coorte retrospectivo para avaliar o Pediatric Obesity Empowerment Model Group Medical Visits como tratamento para obesidade pediátrica em uma comunidade carente, além da redução significativa do escore $\mathrm{z}$ de IMC, encontraram melhora em estresse, nível de exercício, consumo de bebidas açucaradas, consumo de fast food, tempo de televisão e de sono. Fonvig et al., em 1 ano de assistência multidisciplinar de crianças e adolescentes obesos na Dinamarca, além da redução significativa do IMC, observaram a redução da esteatose hepática e esquelética e do volume de tecido adiposo visceral [22].

$\mathrm{O}$ aumento de adiposidade abdominal está fortemente relacionado à resistência periférica à insulina, hipertensão arterial sistêmica, aterosclerose e dislipidemia [23]. No início deste estudo, 85,9\% dos indivíduos atendidos tinham $\mathrm{CC}$ maior que o percentil 90 para a idade e sexo e $68,4 \%$ apresentavam níveis de insulina de jejum maior do que $15 \mu \mathrm{U} / \mathrm{ml} ; 69,8 \%$ índice HOMA maior do que 3,$16 ; 38,8 \% \mathrm{CT}$ maior do que $170 \mathrm{mg} / \mathrm{dl} ; 29,1 \%$ TG maior que $130 \mathrm{mg} / \mathrm{dl}$ e $77,2 \%$ HDL-c menor do que $45 \mathrm{mg} / \mathrm{dl}$. Estes achados se assemelham aos do estudo de Freedman et al., que observaram concentrações mais elevadas de LDL-c, TG e insulina e mais baixas de HDL-c nas crianças com percentil 90 de CC comparadas com as com percentil 10 [24]. 
No estudo de Mastroeni et al., com objetivo de investigar a presença de marcadores de risco para doenças cardiovasculares em adolescentes brasileiros com excesso de peso corporal e com peso normal, os com excesso de peso eram mais propensos a ter alteração de insulina de jejum, índice HOMA e LDL-c, além de níveis reduzidos de HDL-c, semelhante aos indivíduos avaliados no início deste estudo [25].

A redução significativa no $\mathrm{IMC}$ e na $\mathrm{CC}$, a redução no índice HOMA nos primeiros 6 meses e o aumento no HDL-c deste estudo, coincidem com os resultados de Verduci et al., que encontraram redução no escore $\mathrm{z}$ de IMC e nos TG e um aumento no HDL-c. Estes autores também observaram a redução da prevalência de resistência à insulina de $51,8 \%$ para $36,5 \%$ e da síndrome metabólica de $17,1 \%$ para $4,9 \%$, concluindo que intervenções nutricionais e comportamentais podem melhorar o perfil lipídico e a sensibilidade à insulina em crianças obesas e, possivelmente, oferecer benefícios em termos de síndrome metabólica [26].

Parente et al. realizaram um estudo com crianças obesas divididas em dois grupos, dieta e dieta mais prática de atividade física aeróbica, para avaliar as alterações no perfil lipídico. Após 5 meses, nenhuma modificação foi observada nos níveis de CT, LDL-c e TG, porém no grupo dieta mais exercício houve aumento no HDL-c [27]. Estes dados se assemelham aos encontrados neste estudo, mostrando que intervenções com correção de dieta e incentivo à prática de atividade física promovem o aumento do HDL-c em crianças obesas.

No presente estudo, verificou-se que as crianças apresentaram maior redução no escore $\mathrm{z}$ de IMC por idade do que os adolescentes em 1 ano de seguimento, talvez porque os valores de escore $\mathrm{z}$ de IMC por idade eram maiores nesta faixa etária ou porque estavam com excesso de peso há menos tempo, com hábitos errôneos ainda não tão fortemente incorporados quanto os adolescentes. Em função da escassez de publicações sobre este dado, novos estudos são necessários para investigar melhor esta associação.

A epidemia de obesidade infantil tem sido acompanhada por um aumento na prevalência de diabetes mellitus tipo 2 na juventude. Resistência à insulina, estritamente relacionada com o ganho de peso excessivo, é o primeiro passo na patogênese da diabetes mellitus tipo 2. Por esta razão, utilizar um tratamento farmacológico para a obesidade e a resistência à insulina no público infanto-juvenil, visando a prevenção de diabetes mellitus tipo 2 , pode ser considerada uma estratégia racional segundo
Brufani et al. [28]. Estes pesquisadores realizaram uma revisão sistemática sobre a utilização de metformina em crianças e adolescentes obesos não diabéticos para verificar a sua eficácia na redução de peso e na melhora da resistência à insulina. Avaliaram 11 ensaios, em que a metformina foi administrada por 6 a 12 meses, na dosagem de 1000 a $2000 \mathrm{mg} /$ dia, e constataram redução do IMC, além da melhoria na resistência à insulina de jejum, comparando com placebo ou intervenção de modificação de estilo de vida isolada. Porém, neste estudo, a redução no percentual de HOMA não foi significativa e nem houve redução do IMC maior no grupo que utilizou a medicação, semelhante ao encontrado por Park et al. ao fazer uma revisão sistemática com 5 estudos $[28,29]$.

As limitações deste estudo são o fato de ser um estudo retrospectivo, em que dados importantes podem ser perdidos, e o período de acompanhamento relativamente curto, considerando-se que a obesidade infanto-juvenil deve ser tratada de forma contínua e por um longo período, durante todo o crescimento das crianças e adolescentes. Com um acompanhamento maior, as tendências de melhora nos perfis lipídico e glicêmico apresentadas poderiam ser mais bem avaliadas.

Enfim, a estratégia avaliada neste estudo proporcionou uma redução significativa dos níveis de excesso de peso entre as crianças e adolescentes nos 12 meses de acompanhamento em um hospital público de Porto Alegre. Os parâmetros bioquímicos não reduziram na mesma proporção, mas apresentaram uma tendência de melhora. Estes resultados confirmam que estratégias com este perfil, baseadas em aconselhamento, que visam mudanças de comportamento em relação à alimentação e aumento da atividade física, são positivas e seguras e podem ser utilizadas de forma sistemática para combater a atual epidemia de obesidade.

\section{NOTAS}

Parte deste trabalho é resultado de dissertação de mestrado de uma das autoras (LGS) intitulada "Avaliação do impacto de uma estratégia de manejo do excesso de peso infanto juvenil em um hospital público de Porto Alegre" e foi apresentado ao XXV Congresso Brasileiro de Nutrição - CONBRAN, Brasília/DF, abril de 2018, ao XX de Congresso Brasileiro de Nutrologia, São Paulo/ SP, outubro de 2016 e ao II Congresso Brasileiro de Nutrologia Pediátrica - NUTROPED 2016, Belém do Pará/PA, setembro de 2016.

\section{Apoio financeiro}

Este estudo não recebeu apoio financeiro de fontes externas.

Declaração de conflito de interesses

Os autores declaram não haver conflitos de interesses relevantes ao conteúdo deste estudo. 


\section{Contribuições dos autores}

Todos os autores fizeram contribuições substanciais para concepção, ou delineamento, ou aquisição, ou análise ou interpretação de dados; e redação do trabalho ou revisão crítica; e aprovação final da versão para publicação.
Disponibilidade dos dados e responsabilidade pelos resultados

Todos os autores declaram ter tido total acesso aos dados obtidos e assumem completa responsabilidade pela integridade destes resultados.

\section{REFERÊNCIAS}

1. WHO. World Health Organization. Obesity and overweight. [Internet]. [cited 2019 June]. Available from: https://www. who.int/en/news-room/fact-sheets/detail/obesity-and-overweight

2. IBGE. Instituto Brasileiro de Geografia e Estatística. Pesquisa de Orçamentos Familiares 2008-2009: Antropometria e Estado Nutricional de Crianças, Adolescentes e Adultos no Brasil. Rio de Janeiro: IBGE, 2010. [Internet]. [cited 2019 June]. Available from: https://biblioteca.ibge.gov.br/visualizacao/livros/liv45419.pdf

3. Freedman DS, Mei Z, Srinivasan SR, Berenson GS, Dietz WH. Cardiovascular risk factors and excess adiposity among overweight children and adolescents: The Bogalusa Heart Study. J Pediatr. 2007;150(1):12-7. https://doi. org/10.1016/j.jpeds.2006.08.042

4. Carvalho MA, Carmo I, Breda J, Rito AI. Análise comparativa de métodos de abordagem da obesidade infantil. Rev Port Saúde Pública. 2011;29(2):148-56. https://doi.org/10.1016/S0870-9025(11)70019-0

5. Fernandes SP, Conterato EV, Mello ED. Manejo do paciente obeso pediátrico na atenção primária: proposta de uma abordagem terapêutica prática. Rev HCPA. 2012;32(4):461-72.

6. BRASIL. Ministério da Saúde, Secretaria de Atenção à Saúde, Departamento de Atenção Básica. Estratégias para o cuidado da pessoa com doença crônica: obesidade [Internet]. Brasília; 2014 [cited 2019 June]. Available from: http:// bvsms.saude.gov.br/bvs/publicacoes/estrategias cuidado doenca cronica obesidade cab38.pdf

7. Fisberg RM, Marchioni DML, Colucci ACA. Assessment of food consumption and nutrient intake in clinical practice. Arq Bras Endocrinol Metab. 2009;53:617-24. https://doi.org/10.1590/S0004-27302009000500014

8. BRASIL. Ministério da Saúde. Norma Técnica do Sistema de Vigilância Alimentar e Nutricional - SISVAN. Orientações para a coleta e análise de dados antropométricos em serviços de saúde [Internet]. Brasília; 2011. [cited 2019 June]. Available from: http://bvsms.saude.gov.br/bvs/publicacoes/orientacoes_coleta_analise_dados antropometricos.pdf

9. Freedman DS, Serdula MK, Srinivasan SR, Berenson GS. Relation of circumferences and skinfold thicknesses to lipid and insulin concentrations in children and adolescents: the Bogalusa Heart Study. Am J Clin Nutr. 1999; 69:308-17. https://doi.org/10.1093/ajcn/69.2.308

10. Xavier HT, Izar MC, Faria Neto JR, Assad MH, Rocha VZ, Sposito AC, Fonseca FA, dos Santos JE, Santos RD, Bertolami MC, Faludi AA, Martinez TLR, Diament J, Guimarães A, Forti NA, Moriguchi E, Chagas ACP, Coelho OR, Ramires JAF. V Brazilian guidelines on dyslipidemias and prevention of atherosclerosis. Arq Bras Cardiol. 2013;101:1-22. https://doi.org/10.5935/abc.2013S010

11. SBP. Sociedade Brasileira de Pediatria. Obesidade na Infância e Adolescência: Manual de Orientação [Internet]. Rio de Janeiro; 2012. [cited 2018 Nov 3]. Available from: http://www.sbp.com.br/publicacoes/para-a-familia/obesidade-nainfancia-e-adolescencia-manual-de-orientacao-2-edicao-revisada-e-ampliada/

12. Keskin M, Kurtoglu S, Kendirci M, Atabek ME, Yazici C. Homeostasis model assessment is more reliable than the fasting glucose/insulin ratio and quantitative insulin sensitivity check index for assessing insulin resistance among obese children and adolescents. Pediatrics. 2005;115:e500-3. https://doi.org/10.1542/peds.2004-1921

13. Brufani C, Fintini D, Nobili V, Patera PI, Cappa M, Brufani M. Use of metformin in pediatric age. Pediatr Diabetes. 2011;12:580-88. https://doi.org/10.1111/j.1399-5448.2010.00741.x

14. Guimarães LSP, Hirakata VN. Use of the Generalized Estimating Equation Model in longitudinal data analysis. Rev HCPA. 2012;32:503-11.

15. Ludwig DS. Weight loss strategies for adolescents a 14-year-old struggling to lose weight. Jama 2012; 307:498-508. https://doi.org/10.1001/jama.2011.2011 
16. Andela S, Burrows TL, Baur LA, Coyle DH, Gow ML. Efficacy of very low-energy diet programs for weight loss: A systematic review with meta-analysis of intervention studies in children and adolescents with obesity. Obesity Reviews. 2019;20(6). https://doi.org/10.1111/obr.12830

17. Farpour-Lambert NJ, Baker JL, Hassapidou M, Holm JC, Nowicka P, O'Malley G, Weiss R. Childhood obesity is a chronic disease demanding specific health care - a position statement from the Childhood Obesity Task Force (COTF) of the European Association for the Study of Obesity (EASO). Obes Facts. 2015;8:342-9. https://doi. org/10.1159/000441483

18. Zolotarjova J,Velde G, Vreugdenhil ACE. Effects of multidisciplinary interventions on weight loss and health outcomes in children and adolescents with morbid obesity. Obesity Reviews. 2018;19(7). https://doi.org/10.1111/ obr. 12680

19. Most SW, Hojgaard B, Teilmann G, Andersen J, Valentiner M, Gamborg M, Holm JC. Adoption of the Children's Obesity Clinic's Treatment (TCOCT) protocol into another Danish pediatric obesity treatment clinic. Bmc Pediatr. 2015;15:1-8. https://doi.org/10.1186/s12887-015-0332-9

20. Serodio KJ, Berall GB, Flanders DI, Kuk JL. Effectiveness of a publicly funded clinical paediatric weight management program on obesity outcomes. Paediatr Child Health. 2015;20:425-8. https://doi.org/10.1093/pch/ 20.8.425

21. Geller JS, Dube ET, Cruz GA, Stevens J, Bench KK. Pediatric Obesity Empowerment Model Group Medical Visits (POEM-GMV) as treatment for pediatric obesity in an underserved community. Childhood Obesity. 2015;11:638-46. https://doi.org/10.1089/chi.2014.0163

22. Fonvig CE, Chabanova E, Ohrt JD, Nielsen LA, Pedersen O, Hansen T, Thomsen HS, Holm JC. Multidisciplinary care of obese children and adolescents for one year reduces ectopic fat content in liver and skeletal muscle. Bmc Pediatr. 2015;15:1-9. https://doi.org/10.1186/s12887-015-0513-6

23. Coelho M, Oliveira T, Fernandes R. Biochemistry of adipose tissue: an endocrine organ. Arch Med Sci. 2013;9(2):191-200. https://doi.org/10.5114/aoms.2013.33181

24. Freedman DS, Dietz WH, Srinivasan SR, Berenson GS. The relation of overweight to cardiovascular risk factors among children and adolescents: the Bogalusa Heart Study. Pediatrics. 1999;103:1175-82. https://doi.org/10.1542/ peds.103.6.1175

25. Mastroeni SSBS, Mastroeni MF, Gonçalves MC, Debortoli G, Silva NN, Bernal RTI, Adamovski M, Veujelers PJ, Rondó PHC. Cardiometabolic risk markers of normal weight and excess body weight in Brazilian adolescents. Appl Physiol Nutr Metab. 2016;41:659-65. https://doi.org/10.1139/apnm-2015-0632

26. Verduci E, Lassandro C, Giacchero R, Miniello VL, Banderali G, Radaelli G. Change in metabolic profile after 1-year nutritional-behavioral intervention in obese children. Nutrients. 2015;7:10089-99. https://doi.org/10.3390/ nu7125520

27. Parente EB, Guazzelli I, Ribeiro MM, Silva AG, Halpern A, Villares SM. Obese children lipid profile: effects of hypocaloric diet and aerobic physical exercise. Arq Bras Endocrinol Metab. 2006;50:499-504. https://doi.org/ $10.1590 /$ S0004-27302006000300013

28. Brufani C, Crino A, Fintini D, Patera PI, Cappa M, Manco M. Systematic Review of Metformin use in obese nondiabetic children and adolescents. Horm Res Paediatr. 2013; 80:78-85. https://doi.org/10.1159/000353760

29. Park MH, Kinra S, Ward KJ, White B, Viner RM. Metformin for obesity in children and adolescents: a systematic review.(META-ANALYSIS: Reviews/Commentaries/ADA Statements). Diabetes Care. 2009;32:1743-5. https://doi. org/10.2337/dc09-0258 